Clinical Case Report

\title{
Postural Orthostatic Tachycardia Syndrome (POTS): A Case Report
}

\author{
Elnur Tahirović \\ Clinic for Cardiovascular Surgery \\ University Clinical Centre Sarajevo, \\ Sarajevo, Bosnia and Herzegovina \\ Correspondence: \\ elnur.tahirovic@gmail.com \\ Tel.: +38761800269 \\ Fax.: +38735264500
}

Received: August18, 2019

Accepted: September 11, 2019

Key Words: Postural Orthostatic Tachycardia Syndrome • Holter ECG * Non-Pharmacological Interventions.

\begin{abstract}
Objective - The aim of this article is to present a case of POTS, which was diagnosed quickly with Holter ECG and whose symptoms improved promptly and dramatically with non-pharmacological therapy. Case Presentation - A 15-year-old female patient mother was admitted because of unusual episodes of dizziness, syncope with spontaneous recovery and palpitations. Most of these symptoms occurred fairly frequently on standing. The Holter ECG was done with recommendation to perform some activities during recording, such as to stand for ten minutes in the presence of another person and to write symptoms. It was noticed on the Holter ECG that she had increase in heart rate more than 60 beats per minute during these ten minutes followed by symptoms. The patient was diagnosed with POTS and nonpharmacological therapy was advised which was successful. Conclusion - POTS is a disabling condition with heterogeneous and atypical symptoms which mostly affects the young females. Sometimes, a long period of time is necessary to diagnose POTS. Therefore, it is important to diagnose quickly and to start with appropriate therapy which is unique for every POTS patient. Delay in diagnosis and treatment can lead to further disability in patients affected by POTS and their poor quality of life.
\end{abstract}

\section{Introduction}

Postural orthostatic tachycardia syndrome (POTS) is the abnormal increase in heart rate that occurs after sitting or standing, followed by symptoms such as presyncope and syncope, and is frequently associated with hypermobile joints (1). The pathophysiology underlying POTS remains incompletely understood. It is likely to be multifactorial and with multiple pathophysiologic mechanisms and varies in different subgroups of POTS patients (2). POTS predominantly affects women with a female to male ratio of 4.5:1 and with ages ranging from 15 to 50 years $(1,3)$. The patients can be very disabled by their symptoms which are heterogeneous and can range from mild to severe, and vary from day to day which is the reason why POTS is underdiagnosed.

We report a case of POTS, which was diagnosed quickly with Holter ECG and which symptoms improved promptly and dramatically with non-pharmacological therapy.

\section{Case Presentation}

A 15-year-old female patient accompanied by her mother was admitted because of unusual episodes of dizziness, syncope with spontaneous recovery and palpitations. Most of these symptoms occurred fairly frequently on standing. She had symptoms of tiredness, nausea, headache and some gastrointestinal problems such as obstipation, and she did 
not tolerate heat. She said that her legs were heavy and that she noticed excessive sweating of her hands. Recently, she had also concentration problems and with school learning. Most of these symptoms started a few years ago when she was on a vacation with the school. She said that this vacation was very traumatic for her because she was without parents. Prior to this examination, the patient was examined by paediatricians and neurologists and all investigations such as ultrasound, CT of thoracic organs, MRI of the head revealed no significant abnormalities. A physical examination was undertaken, and no abnormalities were noticed except the presence of hypermobile joint and sweaty hands. Her pulse was about $75 \mathrm{bpm}$ and blood pressure 110/80 $\mathrm{mmHg}$. Heart ultrasound showed a structurally normal heart. The Holter ECG was done with recommendation to perform some activities during recording, such as to stand for ten minutes in the presence of another person and to write symptoms. It was noticed on the Holter ECG, when she changed the position of the body, lying to standing, especially after she woke up in the morning, that she had increase in heart rate more than 60 beats per minute during these ten minutes (Fig. 1, 2).

This was followed with symptoms such as dizziness, presyncope symptoms, heavy arms and legs, making it impossible to stand, and giving rise to the need to lie in bed. The patient was diagnosed with postural orthostatic tachycardia syndrome. She was advised to start her treatment with non-pharmacological therapy, to avoid triggers such as prolonged standing, to keep well-hydrated, to increase fluid intake, particularly in the morning, daily minimum 2-3 litres of fluid, salt diet, physical reconditioning and to use compression stockings. Two months later, the patient said she felt better, had less fatigue, palpitations and dizziness, which was also confirmed by her mother, and that the use

17:27:00.0-01 Hours 16 - Artifact

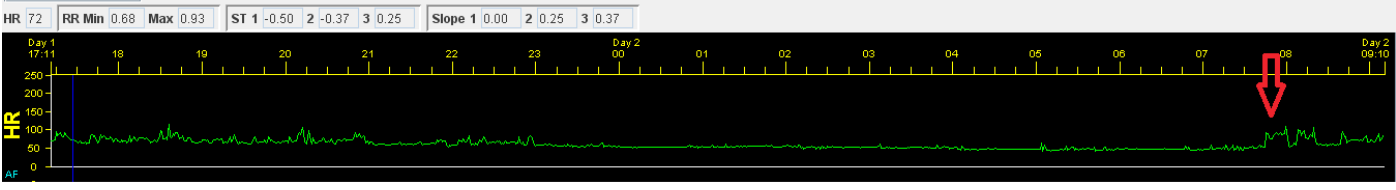

Fig. 1. Heart Rate Trends for 24 Hours. The Red Arrow Shows an Increase in the Heart Frequency After a Change of Body Position in the Morning.

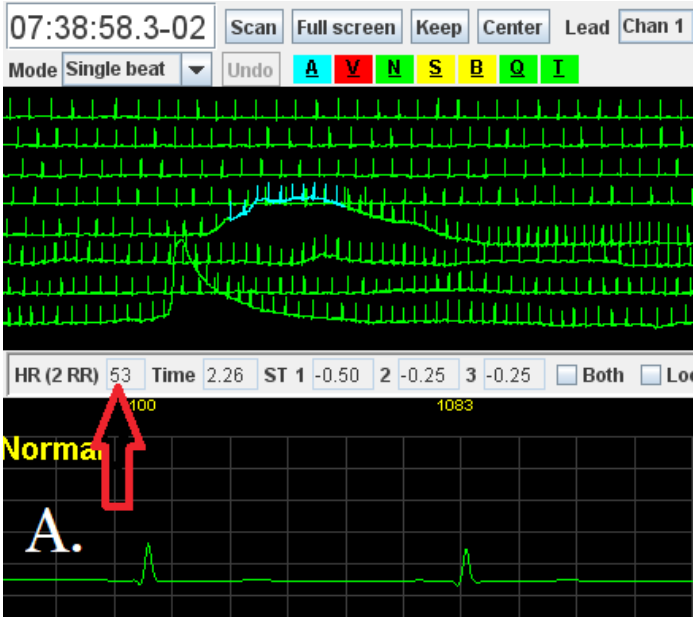

07:51:45.4-02 Scan Full screen Keep Center Lead Chan 1 .

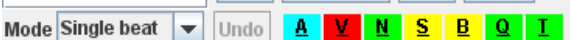

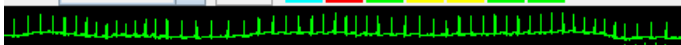

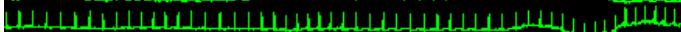

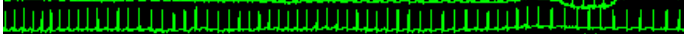

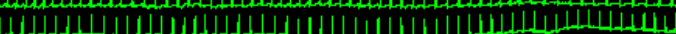

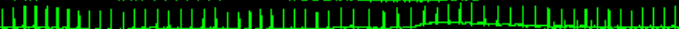

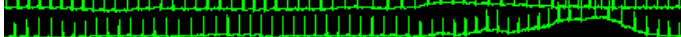

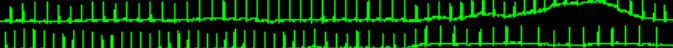

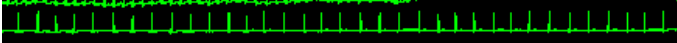

\begin{tabular}{|l|l|l|l|l|l|l|l}
\hline HR (2 RR) 122 & Time 0.98 & ST 1 & -2.12 & 2 & -0.37 & 3 & 0.00
\end{tabular}$\square$ Both $\square$ Lock

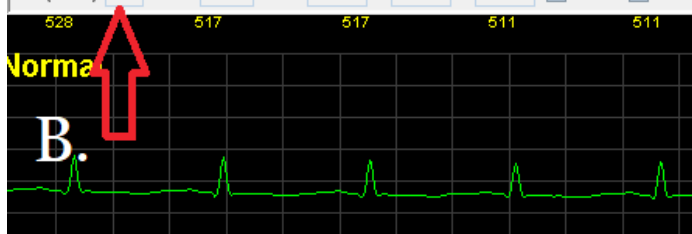

Fig. 2. A: The Red Arrow Shows Heart Rate 53 Beats per Minute in Bed while Lying. B: The Red Arrow Shows Heart Rate 122 Beats per Minute while Standing Followed by Symptoms. 
of compression socks (Fig. 3) contributed to a significant improvement in her problems.

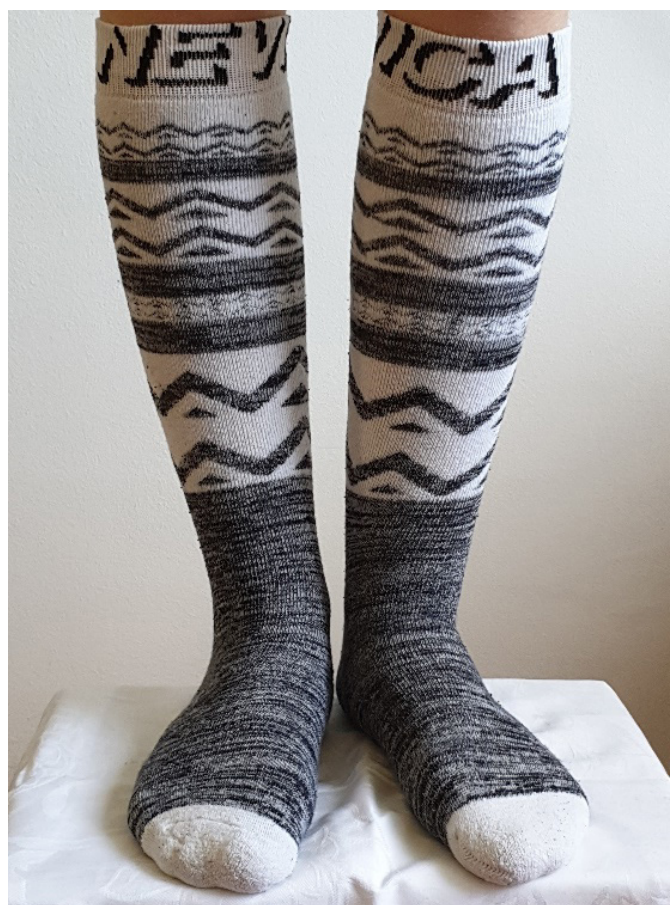

Fig 3. Compressive Stocking Example.

\section{Discussion}

Postural orthostatic tachycardia syndrome is a form of dysautonomia and it is known as soldier's heart, irritable heart, idiopathic orthostatic intolerance, chronic orthostatic intolerance, idiopathic hypovolemia, mitral valve prolapse syndrome (4). It is defined as the presence of orthostatic intolerance associated with a heart rate increase by more than $30 \mathrm{bpm}$ (for the teenagers an increase in $40 \mathrm{bpm}$ ) in an upright posture, within ten minutes of standing, the absence of orthostatic hypotension and duration of symptoms at least six months (2). These findings are necessary for the diagnosis of POTS. The syndrome is frequently associated with hypermobile joints, low blood pressure, chronic fatigue syndrome, mast cell activation disorder, diabetes $(5,6)$. The onset of POTS is preceded by some traumatic event, viral ill- ness, for example with Ebstein-Barr virus, pregnancy, certain medications, surgery, heavy metal exposure, menses, and certain drugs $(7,8)$. Blitshteyn in her article reported six patients with onset of POTS after HPV vaccination (9). Also, Gunning et al. in their study reported that POTS may be an autoimmune disorder and that both sympathetic and parasympathetic nervous system receptors are immune-mediated targets (10).

POTS, as a disorder of the autonomic nervous system, is characterized by heterogeneous, atypical and rather common symptoms which are responsible for "wandering" of the patient from doctor to doctor and searching for a diagnosis. That is the reason why POTS is invisible disease. Symptoms are palpitation, exercise intolerance, fatigue, tremor, lightheadedness, migraine-like headaches, diminished concentration, nausea, obstipation, syncope and near syncope. These symptoms greatly impact the normal functioning with consequent impaired health-related quality of life. Worsening of some of these symptoms while standing up is a main key for the diagnosis of POTS within the differential diagnosis.

There are two major types of POTS, hyperadrenergic and neuropathic. The hyperadrenergic type is present in approximately $50 \%$ of patients with POTS and is familial condition. It is characterised with excessive sympathetic activity, by high levels of noradrenaline in response to standing which causes the orthostatic tachycardia and orthostatic intolerance followed by sweating, tremulousness, anxiety (1115). The second type of POTS, neuropathic is characterized by peripheral denervation of sympathetic nerves leading to impaired peripheral vasoconstriction. This lack of vasoconstriction results in excessive pooling of blood in blood vessels of lower limbs and mesenteric circulation with the reflex tachycardia (7).

For the diagnosis of POTS, with a complete physical examination, it is necessary to perform the head-up tilt table test, the active 
stand test, ECG and Holter ECG monitoring (16). Also, it is necessary to do some laboratory tests to exclude illness such as anemia, pheochromocytoma hypothyroidism or hyperthyroidism, carcinoid or catecholamines on standing from a supine position (2). There is no standard treatment for POTS, and treatment strategies should be based on clinical presentation and treating the underlying pathophysiologic mechanism (11). Therefore, the treatment of POTS is based on non-pharmacological interventions and pharmacotherapy. The pharmacotherapy should be used for the patients who remain symptomatic after three months of optimal lifestyle interventions (11). This therapy is based on use medications for lowering standing heart rate and improving complaints of palpitations such as some $\beta$-blockers in low doses (propranolol, metoprolol) and ivabradine. There are also some vasoconstrictors like $\alpha 1$-adrenergic agonist midodrine and medication that increase blood volume like fludrocortisone which is used for improving peripheral sensitivity of alpha adrenoceptors, fluid and salt retention and desmopresin (2).

But the first therapy for the POTS should be based on non-pharmacological interventions. The lifestyle modifications, which are based on increased fluid intake, particularly in the morning (minimum 2-3 litres of fluid per day), a high salt diet (between 3-10 grams per day) is recommended. This should prevent hypovolemia while hypovolemia seems to play a major role in the most of the patients with POTS $(17,14,18,19)$. Physical reconditioning and resistance training for the lower body is the cornerstone of treatment for patients with POTS (14). Swimming, rowing, recumbent biking progressing on to upright biking and lower limb resistance training is beneficial or even curative for some POTS patients. In was found in POTS registry that after three months of training $71 \%$ patients no longer met objective criteria for POTS and were considered to be in remission (20). Also, it is recommended to elevate the head of the bed off the ground for $10-50 \mathrm{~cm}$ in order to increase circulating plasma and blood volume. For the reduction of venous pooling, it is recommended to use compression garment and stocking which decreased venous pooling, increased systolic blood pressure, attenuated the reductions in stroke volume and cardiac output (21).

In our case report, there are two main findings that are important to notice. The first is that this patient could not be diagnosed for the longer period because of heterogeneous and atypical symptoms. Indeed, according to the available literature, the average time to diagnose POTS is 5 years and 11 months (22). In our case there were situations when doctors told the patient's mother that her child is healthy and that everything was "in her head". But that is because POTS is an invisible illness. It is important to raise awareness of POTS and to reduce the period of seeking a diagnosis of POTS and begin treatment as soon as possible. The second thing is that the treatment with non-pharmacological interventions was very successful, especially when the patient started to use the compression stockings which, according to her mother, contributed to a dramatic improvement in symptoms and improved patient functioning. It is recommended to use the compression stockings during daily activities with 30 to $40 \mathrm{~mm} \mathrm{Hg}$ of pressure (17). Fu et al. in their study concluded that non-drug therapy is superior to $\beta$-blockers and therefore exercise and non-pharmacological treatment should be considered as soon as possible in the treatment of POTS (23).

\section{Conclusion}

POTS is a disabling condition with heterogeneous and atypical symptoms which mostly affects the young females. Sometimes a long period of time is necessary to diagnose POTS. Therefore, it is important to diagnose quickly 
and to start with appropriate therapy which is unique for every POTS patient. Delay in diagnosis and treatment can lead to further disability in patients affected by POTS and their poor quality of life.

Conflict of Interest: The author declares that he has no conflict of interest.

\section{References}

1. Low PA, Opfer-Gehrking TL, Textor SC, Benarroch EE, Shen WK, Schondorf R, et al. Postural tachycardia syndrome (POTS). Neurology. 1995;45:S19-S25.

2. Wells R, Spurrier AJ, Linz D, Gallagher C, Mahajan R, Sanders P, et al. Postural tachycardia syndrome: current perspectives. Vasc Health Risk Manag. 2017;14:1-11.

3. Benarroch EE. Postural tachycardia syndrome: a heterogeneous and multifactorial disorder. Mayo Clin Proc. 2012;87:1214-25.

4. Kavi L, Gammage MD, Grubb BP, Karabin BL. Postural tachycardia syndrome: multiple symptoms, but easily missed. Br J Gen Pract. 2012;62:286-7.

5. Beighton P, Solomon L, Soskolne CL. Articular mobility in an African population. Ann Rheum Dis. 1973;32:413-8.

6. Anjum I, Sohail W, Hatipoglu B, Wilson R. Postural Orthostatic Tachycardia Syndrome and Its Unusual Presenting Complaints in Women: A Literature Minireview. Cureus. 2018;10:e2435.

7. Abdulla A, Rajeevan T. Reversible postural orthostatic tachycardia syndrome. World J Clin Cases. 2015;16:3(7):655-60.

8. Pramya N, Puliyathinkal S, Sagili H, Jayalaksmi D, Reddi Rani P. Postural orthostatic tachycardia syndrome complicating pregnancy: a case report with review of literature. Obstet Med. 2012;5(2):83-5.

9. Blitshteyn S. Postural tachycardia syndrome following human papillomavirus vaccination. Eur J Neurol. 2014;21(1):135-9.

10. Gunning WT 3rd, Kvale H, Kramer PM, Karabin BL, Grubb BP. Postural Orthostatic Tachycardia Syndrome Is Associated With Elevated G-Protein Coupled Receptor Autoantibodies. J Am Heart Assoc. 2019 Sep 17;8(18):e013602.

11. van der Zalm T, Alsma J, van de Poll SWE, Wessels MW, Riksen NP, Versmissen J. Postural orthostatic tachycardia syndrome (POTS): a common but unfamiliar syndrome. Neth J Med. 2019;77(1):3-9.

12. Benarroch EE. Postural tachycardia syndrome: a heterogeneous and multifactorial disorder. Mayo Clin Proc. 2012;87:1214-25.

13. Arnold AC, Ng J, Raj SR. Postural tachycardia syndrome - Diagnosis, physiology, and prognosis. Auton Neurosci. 2018;215:3-11.

14. Sheldon RS, Grubb BP 2nd, Olshansky B, et al. 2015 heart rhythm society expert consensus statement on the diagnosis and treatment of postural tachycardia syndrome, inappropriate sinus tachycardia, and vasovagal syncope. Heart Rhythm. 2015;12:e41-63.

15. Grubb BP. Postural tachycardia syndrome. Circulation. 2008; 27:117(21):2814-7.

16. Kirbiš M, Grad A, Meglič B, Bajrović FF. Comparison of active standing test, head-up tilt test and 24-h ambulatory heart rate and blood pressure monitoring in diagnosing postural tachycardia. Funct Neurol. 2013;28:39-45.

17. Raj SR. Postural tachycardia syndrome (POTS). Circulation. 2013;127:2336-42.

18. Ponnusamy V, Owens AP, Purkayastha S, Iodice V, Mathias CJ. Orthostatic intolerance and autonomic dysfunction following bariatric surgery: A retrospective study and review of the literature. Auton Neurosci. 2016;198:1-7.

19. Z'Graggen WJ, Hess CW, Humm AM. Acute fluid ingestion in the treatment of orthostatic intolerance - important implications for daily practice. Eur J Neurol. 2010;17:1370-6.

20. George SA, Bivens TB, Howden EJ, Saleem Y, Galbreath MM, Hendrickson D, et al. The international POTS registry: Evaluating the efficacy of an exercise training intervention in a community setting. Heart Rhythm. 2016;13(4):943-50.

21. Stenger MB, Brown AK, Lee SM, Locke JP, Platts $\mathrm{SH}$. Gradient compression garments as a countermeasure to post-spaceflight orthostatic intolerance. Aviat Space Environ Med. 2010;81(9):883-7.

22. Dysautonomia International [homepage on the Internet] [cited 2019 Aug 08]. Available from: https://www.dysautonomiainternational.org/pdf/ PhysicianPatientInteractionInPOTS.pdf.

23. Fu Q, Vangundy TB, Shibata S, Auchus RJ, Williams $\mathrm{GH}$, Levine BD. Exercise training versus propranolol in the treatment of the postural orthostatic tachycardia syndrome. Hypertension. 2011;58(2):167-75. 